\title{
A Comparative Life Cycle Assessment of Single-Use Fibre Drums Versus Reusable Steel Drums
}

\author{
Marco Raugei ${ }^{1, *}$, Pere Fullana-i-Palmer ${ }^{1}$, Rita Puig $^{2}$, Alejo Torres $^{3}$ \\ ${ }^{1}$ Environmental Management Research Group (GiGa) \\ Escola Superior de Comerç Internacional - Universitat Pompeu Fabra \\ Pg. Pujades 1, 08003 Barcelona, Spain \\ ${ }^{2}$ Grup Igualada Recerca (GIR) \\ Escola Universitària d'Enginyeria d'Igualada - Universitat Politècnica de Catalunya \\ Pl. del Rei 1, 08700 Igualada, Spain \\ ${ }^{3}$ Environment, Health and Safety Department, Grupo URIACH \\ Polígono Industrial Riera de Caldes, Avinguda Camí Reial 51-57 \\ 08184 Palau Solita i Plegamans (Barcelona)
}

\begin{abstract}
This paper deals with a comparative analysis of two different packaging and transport scenarios, which exemplifies the implications of choosing between single-use and reusable packaging. In particular, transport of a batch of chemicals by means of disposable fibre drums vs. reusable steel drums is investigated from a life cycle perspective, and the associated environmental impact in terms of Global Warming Potential, Acidification Potential, Gross Energy Requirement and solid waste generation is assessed. Results prove beyond reasonable doubt that, even in the case of durable packaging containers requiring the use of comparatively energy-intensive materials for their production, the reuse scenario is characterized by lower environmental impact indicators across the board, and as such is the most advisable and environmentally sound option.
\end{abstract}

Key words: LCA; packaging; transport; single-use; reuse.

\footnotetext{
* Corresponding author: Environmental Management Research Group (GiGa), Escola Superior de Comerç Internacional - Universitat Pompeu Fabra, Barcelona, Spain. E-mail: marco.raugei@admi.esci.es ; telephone: (+ 34) 932954710
} 


\section{Introduction}

In recent years, waste disposal capacity has turned more and more into a limited resource in most countries, and increasing concern has been voiced about the need to curb down on unnecessary waste production caused by single-use packaging. On top of that, packaging is often also responsible for a considerable fraction of the energy and material requirements associated to the life cycle of a product, and is thus also detrimental to the latter's overall sustainability, both in terms of resource depletion and of the associated emissions. Besides reducing the sheer amount of packaging per unit of product (e.g. by means of large batch delivery), the two most effective strategies to address these ever more impending issues appear to be: on the one hand promoting the reuse of the packaging containers wherever viable, and on the other hand making sure that the containers themselves are fabricated out of recyclable materials, so that a savings of primary resources can be achieved at the end of their useful lifetime. The current CEN standard on Life Cycle Assessment (LCA) and packaging also provides similar recommendations [1].

The majority of the relatively few comparative studies in the scientific literature have so far been concerned with reuse and / or recycling scenarios of one given type of packaging [2; 3]. In most such cases, results have shown that implementing either strategy (or, when possible, both at the same time) invariably leads to a win-win situation, where lower environmental impact may be achieved both in terms of reduced waste generation and reduced demand for primary resources. It is however interesting and relevant to extend the scope of the analysis to also include comparisons between substantially different packaging options. In fact, in order to effectively replace a singleuse container with a more durable one which may be used multiple times, the latter may have to be manufactured out of much more energy-intensive materials than the former. Furthermore, the more durable packaging container will also often be heavier than its single-use equivalent, thereby requiring additional fuel for its transport. In these cases, it may therefore not look straightforward anymore whether the aforementioned advantages of the reuse scenario in terms of reduced environmental impact still hold.

In this paper, the authors present one such case, where two scenarios are compared and discussed. The product being transported is in both cases a batch of raw pharmaceutical chemicals, which is moved by lorry over a distance of $8.5 \mathrm{~km}$, from the 
chemical production plant to the pharmaceutical plant. The two considered packaging options are:

Scenario 1 - a number of single-use disposable fibre drums, which are landfilled at the end of each one-way trip;

Scenario 2 - a number of reusable steel drums having an equivalent capacity, which are transported back empty to the chemical production plant and washed for reuse several times, before finally being disposed of.

\section{Method}

The analysis is performed in accordance to the recently updated ISO standards on LCA $[4 ; 5]$. The goal of the analysis is to compare the environmental performance of the two packaging and transport options, in terms of solid waste generation as well as of selected life cycle impact assessment indicators. Furthermore, by virtue of its general applicability, besides producing accurate results for this specific case study, the present analysis is also expected to provide a qualitative indication of the extent to which replacing single-use packaging with more energy- and material-intensive multiple-use packaging may be considered an environmentally sound strategy.

From a practical standpoint, the analysis was carried out making use of the $\mathrm{GaBi} 4$ LCA software package [6] and of its integrated Professional Database. Table 1 lists the main assumptions related to the two investigated scenarios. Scenario 1 corresponds to a former transport practice and the relative data were obtained in a previous research project [7], while scenario 2 depicts the current situation. All the relevant updated inventory information was provided by the actual pharmaceutical firm on which the case study is based [URIACH, personal communication 2008].

Figures 1 and 2 illustrate the block diagrams of the production processes for one disposable and one reusable drum, respectively. Virgin (i.e. non-recycled) primary materials are assumed to be employed in both cases. It should be noted that in the case of the reusable drum, however, only a fraction of the necessary inputs for its production is allocated to the finished product (i.e. 1/200), which reflects the assumed average number of times that the drum is used over its life time.

Figures 3 and 4 then illustrate the corresponding transport scenarios. In a similar way as discussed above, in the case of reusable drums, their end-of-life transport to disposal (landfill) is modelled as discounted over the number of uses. Assuming the reusable containers to be landfilled at the end of their useful life can be regarded as a 
"worst case" scenario for the reusable container option. Another possibility is clearly that of recycling, and indeed it would be quite possible here because of the economic value of steel. In this latter case, applying the open-loop recycling model, no end-of-life emissions would be taken into account within the boundaries of the analyzed system, since these would instead be entirely attributed to those other product systems which then make use of the steel scraps in the form of recycled steel [8].

As regards the Life Cycle Impact Assessment phase, the choice was made to employ the widely accepted CML2001 method [9], focussing on two among the most commonly reported impact indicators, i.e. Global Warming Potential (GWP) and Acidification Potential (AP). Moreover, the overall Gross Energy Requirement (GER) of the two scenarios, expressed in terms of higher heating value (HHV) of primary energy supply, is provided as an aggregated indicator of environmental impact from the point of view of the depletion of non-renewable energy resources. Last but not least, the sheer amount of solid waste delivered to the landfill is itself taken as a proxy indicator of "un-sustainability", given the rapidly diminishing capacity of the existing landfills worldwide, and the often greater and greater difficulty in finding suitable sites for new ones. This is especially the case in Catalonia, where a replacement site for the Garraf landfill for household waste (which was brought to a complete halt on 1 January 2007) has not yet been found, and the industrial waste landfill in Vaquerisses is currently being used for household waste disposal too, putting the industrial waste landfilling capacity at risk. All such indicators are calculated on the basis of one functional unit of transported goods, which in the case of the present case study corresponds to a batch of $540 \mathrm{~kg}$ of chemicals.

ISO standard 14044 [5] explicitly states that a comparative LCA intended to be disclosed to the public should never present results in terms of aggregated indicators, whereby the calculated potential impacts in different categories are combined to produce a single number. Accordingly, the results of this study are presented in disaggregated form, thus allowing for a more articulated interpretation stage, where any possible controversial points are dealt with individually, and no loss of information is suffered.

\section{Results}

Figure 5 illustrates the results of the comparative study in terms of GWP, AP, GER and amount of solid waste delivered to landfill. 
A very large difference can be seen in favour of the second scenario. In particular, in both the considered impact categories that are related to airborne emissions (GWP and AP), the indicators for the deployment of reusable steel drums only reach approximately $3-3.5 \%$ of the corresponding values for single-use fibre drums, i.e. $1.4 \mathrm{~kg} \mathrm{CO}$-eq and $0.0058 \mathrm{~kg} \mathrm{SO}_{2}$-eq vs. $39 \mathrm{~kg} \mathrm{CO}_{2}$-eq and $0.19 \mathrm{~kg} \mathrm{SO}_{2}$-eq, respectively. A similar result is also obtained with regards to Gross Energy Requirement, where the use of the steel drums allows a 93\% reduction, from $262 \mathrm{MJ}$ down to $19 \mathrm{MJ}$ of primary energy. Last but not least, the total amount of solid waste expressed per functional unit of transported goods also drops from $30 \mathrm{~kg}$ in scenario 1 to $0.27 \mathrm{~kg}$ in scenario 2.

\section{Discussion}

Besides illustrating the improvements achieved in a practical case study from the point of view of different impact categories, the results produced in this study may be taken to clearly indicate a more general marked advantage of reusable transport containers vs. single-use ones. It is especially interesting to underline that this was shown to be the case when comparing the use of relatively "cheap" and non-energy intensive cardboard drums to steel drums that are over three times as heavy, and require over thirty-seven times as much energy for their production. In fact, those characteristics which might appear at first sight as drawbacks (i.e. sturdier construction thanks to more durable materials, and consequently heavier weight and higher energy demand per unit), often turn out to be the very same ones that enable the container's useful service time to be dramatically extended, thereby amply offsetting any initially negative effect in terms of environmental impact. It should be noted that this appears to be especially true for industrial containers, where a large mass of product is transported per individual container. For consumer goods, results in terms of GER and GWP may be not as clear cut, since the relative weight of the packaging may be considerably larger. A previous study focussing on comparing reusable plastic containers to corrugated cardboard container for food produce, however, seems to confirm that in most cases at least a moderate advantage still holds for the reusable containers [10].

One important specific source of impact that generally applies to all cardboard containers, such as those employed in the first scenario considered here, is the considerable emissions of methane that occur when the cardboard is disposed of and anaerobically decomposed in landfills. Since methane has a much higher global warming potential than carbon dioxide (its characterization factor in terms of GWP100 
is 23 times that of $\mathrm{CO}_{2}$ ), the positive contribution of these emissions to global warming amply exceeds the carbon-neutrality due to the cardboard being manufactured out of renewable fibre. In fact, over one half (54\%) of the GWP associated to scenario 1 is associated to the disposal of the single-use drums.

As far as the robustness and general applicability of our findings are concerned, a sensitivity analysis has shown that the most relevant parameter that ends up affecting the comparison in an almost exponential manner is the number of times that the containers are used before finally being disposed of. In the specific case study presented here, the impact of the reusable steel drum scenario rapidly drops below that of the single-use fibre drums as the average number of uses for each drum is increased (Figure 6). In fact, reusing the steel drums as few as four times would suffice from the point of view of equating the GWP of the two transport options, and similarly low numbers of minimum uses are also found for the other considered impact indicators. Clearly, such "parity" cases are rather extreme and unrealistic, as there is no practical reason why a well-manufactured steel drum should be disposed of after so few trips.

The dependency on the second parameter in order of relevance, i.e. the transport distance between the two plants, is almost linear. As shown in Figure 7, under the basecase assumption of 200 uses for each drum, it would take a distance of over $200 \mathrm{~km}$ for a break-even point to be reached when considering the impact in terms of GER, and as much as almost $500 \mathrm{~km}$ in terms of GWP and AP. While such large distances cannot be ruled out when dealing with some steps of the often international supply chain of many industrial processes, they clearly do not apply to typical local transport scenarios between two production plants as those analyzed here.

Additionally, other differences are also to be expected when considering the transport of large batches of goods over very long distances. Firstly, bigger containers with a favourably lower container weight / transported goods weight ratio would be employed. This would reduce the relative importance of the containers themselves vs. the amount of fuel used in both scenarios, thus making the very choice between the two packaging options less relevant. Secondly, it may be assumed that a better packaging efficiency would be reached than in the case study analyzed here, where for instance the necessity to use an integer number of drums for the transport of a given batch of chemicals resulted in less-than-optimal filling of the disposable fibre drums (i.e. six 100 kg drums for $540 \mathrm{~kg}$ of chemicals). Going back to our case study, however, it should be noted that the improvement in the overall environmental performance of scenario 1 
which would result from assuming optimal filling of the fibre drums could at best be expected to be in the order of $10 \%$ (by coarsely assuming the impact to be directly proportional to the drum / content weight ratio, and disregarding all other contributions such as that of the transport fuel). Clearly, such limited improvement would not alter the outcome of the comparison between the two scenarios in any significant way.

In the end, our study has shown beyond reasonable doubt that, even in the case of durable packaging containers requiring the use of comparatively energy-intensive materials for their production, the reuse scenario is characterized by lower environmental impact indicators across the board, and as such is the most advisable and environmentally sound option, at least for transport over short to medium distances.

\section{Acknowledgements}

The authors wish to acknowledge the support provided by URIACH by means of the provision of detailed information on their packaging and transport practices. URIACH also acknowledges the financial support given by the Spanish Government, through the Initiative for Technology, Safety and Quality Support in Industry [7], to develop the research project which delivered the data used for the scenario 1 calculations, and Ms. Marta Vallès and Ms. Natalia Anguera, who participated in that project together with one of the authors (Pere Fullana-i-Palmer).

\section{References}

1. CEN, CEN/CR 13910 - Packaging. Report on criteria and methodologies for life cycle analysis of packaging. Comité Européen de Normalisation (CEN), 2001 http://www.cen.eu/cenorm/homepage.htm

2. Ross s., Evans D. The environmental effect of reusing and recycling a plastic-based packaging system. Journal of Cleaner Production, 2003; 11(5):561-571

3. Gatti J.B., Queiroz G.D., Garcia E.E.C. Recycling of aluminum can in terms of life cycle inventory (LCI). International Journal of Life Cycle Assessment, 2008; 13(3) :219-225

4. ISO 14040:2006 - Environmental Management. Life Cycle Assessment. Principles and Framework. International Organization for Standardization, 2006. http://www.iso.org/iso/home.htm 
5. ISO 14044:2006 - Environmental Management. Life Cycle Assessment. Requirements and Guidelines. International Organization for Standardization, 2006. http://www.iso.org/iso/home.htm

6. GaBi Software Professional, 2007. http://www.gabi-software.com/

7. ATYCA, Life cycle assessment of a pharmaceutical speciality (ATYCA, E681/1999), project by URIACH financed by the Spanish Government through the Initiative for Technology, Safety and Quality Support in Industry, 1999.

8. Baumann, H., Tillman, A.M. The Hitch Hiker's guide to LCA. An orientation in life cycle assessment methodology and application. Studentlitteratur. Sweden, 2004.

9. Guinée, J. (editor) Life cycle assessment: An operational guide to the ISO standards, Leiden University, centre for Environmental Sciences, 2001; 601 pp. (available at http://www.leidenuniv.nl/cml/ssp/index.html)

10. Singh S. Paul, Chonhenchob V., Singh J. (2006). Packaging Technology and Science 2006; 19:279-293 
Scenario 1

Transported batch

Drum type

Drum capacity

Drum weight

n. of drums per batch

Transport distance

(full drums, A to B)

n. of uses per drum

Transport distance

(empty drums, B to A)

Transport distance

(end of life, to landfill)
$540 \mathrm{~kg}$

Disposable (fibre)

$100 \mathrm{~kg}$

$5 \mathrm{~kg}$

6

$8.5 \mathrm{~km}$

1

$10 \mathrm{~km}$
200

Scenario 2

$540 \mathrm{~kg}$

Reusable (steel)

$180 \mathrm{~kg}$

$18 \mathrm{~kg}$

3

$8.5 \mathrm{~km}$

$8.5 \mathrm{~km}$

$10 \mathrm{~km}$

Table 1. Main assumption relative to the two analysed packaging and transport scenarios. $\mathrm{A}=$ chemical production plant; $\mathrm{B}=$ pharmaceutical plant. Sources: [7; URIACH, personal communication 2008]. 
Figure captions:

Figure 1. Block diagram of the production process of one disposable fibre drum.

Figure 2. Block diagram of the production process of one reusable steel drum.

Figure 3. Block diagram of transport scenario 1.

Figure 4. Block diagram of transport scenario 2.

Figure 5. LCIA results in terms of Global warming Potential, Acidification Potential, Gross Energy Requirement and Waste generation.

Figure 6. Variation of environmental impact of scenario 2 relative to scenario 1 for each doubling of number of uses per container.

Figure 7. Variation of environmental impact of scenario 2 relative to scenario 1 for each quadrupling of transport distance. 